${ }^{3}$ Department for Family Medicine, Medical Faculty Sarajevo, Sarajevo; ${ }^{4}$ Pediatrics Surgery, Clinical Center Mostar, Mostar; ${ }^{5}$ Department for Pharmacology, Pharmaceutical Faculty Sarajevo, Sarajevo; ${ }^{6}$ Cardiology Department, Cantonal Hospital Zenica, Zenica; ${ }^{7}$ Pediatrics Department, Pediatrics Clinic, Tuzla; ${ }^{8}$ Pediatrics Department, Clinical Medical Center, Sarajevo, Bosnia-Herzegovina

Introduction Migration refers to the movement of persons or children from an origin place to a destination place across some predefined, political boundary. Since the 1995s after war, Bosnia and Herzegovina has continued being a country of mass children immigration from Sandjak, Kosovo, Serbia, Monte Negro and sporadic immigration from China.

Methods The presence of tuberculosis disease in the Immigrants children or foreign-born child should prompt the pediatricians to collect appropriate specimens to recover an organism. We conducted a secondary data analysis focusing on immigrants children sampled in the 1995 through 2010 versions of the National Bosnian Children Health Records Survey.

Results The increase in tuberculosis among Gypsy children in Sarajevo coincided with similar increases in immigration into Bosnia and Herzegovina. Medical records were available for review to assess adequately potential missed opportunities to prevent tuberculosis in children from Sandjak in only $1.5 \%$ of cases and Gypsies in 33\% cases. Most children with drug-resistant tuberculosis were Gypsy (18.1\%) or Chinese Asian (11.2\%), and $16.4 \%$ of children or their parents were from a Bosnia and Herzegovina regions in which tuberculosis is highly endemic as Sarajevo Canton mountain area.

Conclusions Pediatricians should be aware of the special health problems as tuberculosis for which immigrant children are at risk. Immigration poses unique stresses on children and families. There were no significance difference between incidence of tuberculosis and resistence on therapy between children from Sandjak and Bosnia but that differences were higher in case of Gypsies children.

\section{TOTAL PARENTERAL NUTRITION AS A SOURCE OF OXIDANT LOAD LEADING TO BRONCHOPULMOANRY DYPLASIA}

doi:10.1136/archdischild-2012-302724.0610

${ }^{1}$ ISI Mohamed, ${ }^{2 T}$ Rouleux, ${ }^{2,3} \mathrm{JC}$ Lavoie. ${ }^{1}$ Pediatrics, Division of Neonatology, University of Montreal, Sainte-Justine Hospital; ${ }^{2}$ Centre de Recherche; ${ }^{3}$ Pediatrics- Neonatology, Universite de Montreal - CHU Sainte-Justine, Montréal, OC, Canada

Background Immaturity of antioxidant defense coupled with oxidant load is suspected to induce the development of bronchopulmonary dyplasia (BPD) in premature infants. Peroxide load from total parenteral nutrition (TPN) is associated with oxidative stress in this population. We hypothesize that the oxidative stress and, consequently, the severity of $\mathrm{BPD}$, both increase in function of duration of TPN infusion.

Objective To document the relation between the duration of TPN as well as the redox potential of glutathione measured in blood and the severity of BPD.

Design/methods GSH and GSSG from whole blood sampled at 36 weeks of corrected age in 51 infants less than 29 weeks of gestational age, were measured by capillary electrophoresis in order to calculate the redox potential (Nernst equation). Severity of BPD was classified according to NICH guidelines (Job and Bancalari, 2001). Means (s.e.m). ( $n=5-21$ per group) were compared by ANOVA.

Results The duration of TPN in days was strongly associated $(p<0.001)$ with the severity of BPD. A logestic regression model confirmed the independent effect of TPN.

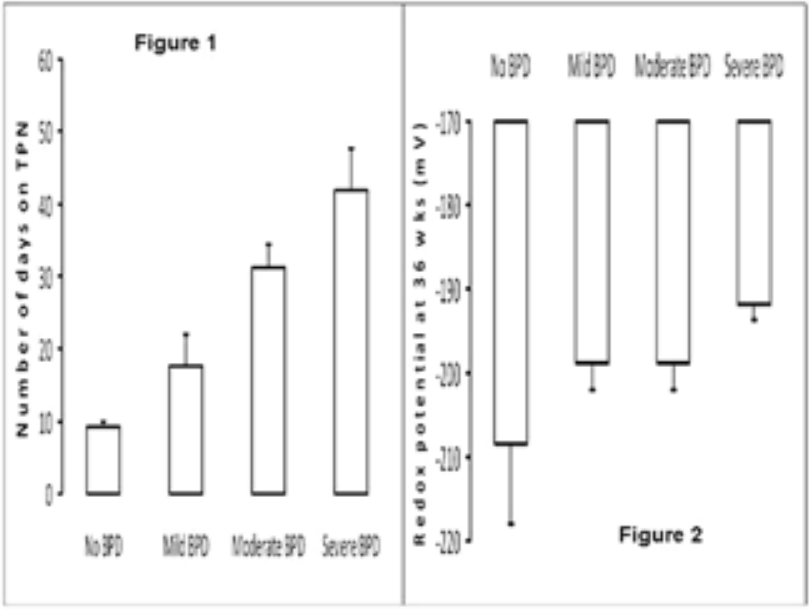

Abstract 610 Figure 1 TPN, redox potential and BPD

Conclusions The duration of the oxidant load from TPN exacerbates the oxidative stress in preterm infants as observed with the more oxidized status of the redox potential in infants having received TPN for a longer time. The strong relation between severity of BPD and duration of TPN could be explained by this oxidative stress generated by the TPN

\section{OESOPHAGEAL ATRESIA AND ASSOCIATED ANOMALIES}

doi:10.1136/archdischild-2012-302724.0611

P Desai, J Morris, L Segal. St George's Hospital NHS Trust, London, UK

Background In previous studies the incidence of associated congenital anomalies for infants with oesophageal atresia(OA) ranges between $40-57 \%$. OA mostly associated with Tracheoesophageal fistula is a part of VACTERL association (Vertebral anomalies, Anal atresia, Cardiovascular anomalies, Tracheoesophageal fistula, Renal/ or radial anomalies, Limb defects).

Aim To determine the incidence of associated anomalies in babies with oesophageal atresia.

Methods 32 babies treated for oesophageal atresia at our regional surgical neonatal unit between the period February 2007 to February 2012 were included in the study.

Results Out of 32 babies, 17 were male and 15 were female infants with gestation ranging from 31-40 weeks. 10 babies (31\%) were born in-utero and 22 babies were transferred from local neonatal units. Birth weights ranged between $1380 \mathrm{~g}-4300 \mathrm{~g}$.

In total 15 babies (47\%) had some form of Cardiac anomalies with 5 babies either having Atrial septal defect or Ventricular septal defect and 8 babies having a patent ductus arteriosus (PDA).

3 babies ( $9 \%$ ) had associated anorectal anomalies.

In total 10 babies (31\%) had associated anomaly excluding isolated PDA and 16 babies (50\%) if isolated PDA were included.

Conlcusion Association of anomalies with Oesophageal anomalies is well documented in literature. It is important to identify vertebral anomalies early for follow up as the risk of scoliosis is 13 fold after repair of $O A$ in relation to general population. This small study showed incidence of $50 \%$ for babies with $\mathrm{OA}$ to be associated with other anomalies and supports current literature.

\section{FEASBILITY STUDY USING FACIAL ANALYSIS SOFTWARE TO DOCUMENT FACIAL FEATURES ASSOCIATED WITH FETAL ALCOHOL SYNDROME IN NEWBORN INFANTS}

doi:10.1136/archdischild-2012-302724.0612 
'K Thorup, 'V Monk, 'E Gourlay, ${ }^{2 N}$ Aiton. 'Brighton and Sussex Medical School; ${ }^{2}$ Brighton and Sussex University Hospitals NHS Trust, Brighton, UK

Background Fetal Alcohol Syndrome (FAS) comprises a triad of growth impairment, central nervous system dysfunction and characteristic facial features. Diagnosis is complex and often not recognised at an early age. The three facial features: short palpebral fissures, smooth philtrum and thin upper lip, are unique to FAS. Clinical examination is inherently subjective and apart from palpebral fissure length, minimal reference data is available in neonates. Establishing a standardised method and normal range would promote an objective assessment. Earlier diagnosis would enable earlier effective interventions.

Methods Standardised digital facial photographs were taken of normal term Caucasian neonates. Mothers completed anonymous questionnaires about alcohol consumption during pregnancy. Photographs were assessed using Facial Analysis Software to obtain values for palpebral fissure length (PFL) and upper lip circularity (LC). Upper lip thinness and philtrum smoothness were ranked according to 5-point Likert Scale.

Results 29 infants were studied, 17 male: 12 female. Mean gestational age 40.3 weeks (range 37.1-42.3), mean weight 3556g. 23 (79\%) had no prenatal alcohol exposure whilst 6 had minimal exposure (1-2units/week). PFL measurements could be obtained from 21 photographs (72\%) with mean of $15.6 \mathrm{~mm}$ (range 13.7-18.7mm). Upper lip and philtrum values could be determined in 24 (83\%). Mean LC was 57.21 (range 31.4-128.2). Mean rank scores for upper lip and philtrum were both 3 .

Conclusion It has been possible to gain measurements of facial features in just over $3 / 4$ of neonates studied, showing the feasibility of this technique in this age-group. Further results are needed to establish reference ranges.

\section{CONGENITAL DIAPHRAGMATIC HERNIA SURVEILLANCE IN IRELAND}

doi:10.1136/archdischild-2012-302724.0613

1,2J Chukwu, 3,4,5EJ Molloy. 'Clinical Research Unit, National Children's Research Centre; ${ }^{2}$ Molecular \& Cellular Therapeutics, Royal College of Surgeons; ${ }^{3}$ Department of Neonatology, Our Lady's Children's Hospital, Crumlin, Dublin 12; ${ }^{4}$ School of Medicine amd Medical Sciences, University College Dublin; ${ }^{5}$ Neonatology, National Maternity Hospital, Holles Street, Dublin, Ireland

Introduction The incidence of Congenital Diaphragmatic Hernia $(\mathrm{CDH})$ varies from 1 in 2,000 to 15,000 births per annum. Due to the legislation prohibiting termination of any pregnancy in the Republic of Ireland (ROI) we hypothesised that the incidence would be different to international reports. There is no mandatory reporting of $\mathrm{CDH}$ cases in the ROI and Northern Ireland (NI).

Aims To determine the true incidence of $\mathrm{CDH}$ in the ROI and NI. Methods Reporting of $\mathrm{CDH}$ cases to the Irish Paediatric Surveillance Unit (IPSU) based in Dublin started in January 2010. Doctors reported new cases to IPSU using the pro-forma provided. Details of the reported cases were collected using a detailed $\mathrm{CDH}$ questionnaire.

Results 23 cases were reported to the IPSU within the 2 year period consisting of 10 cases in 2010 and 13 in 2011; 8 cases from NI and 15 cases from ROI. The incidence rate in the 2 years using IPSU figures was 0.12 per 1000 live births per annum. The questionnaire was completed in 12 cases. 11/12(92\%) made it to the surgical centres. However the Hospital in Patient System record in one of the three surgical centres indicated that only $6 / 15(40 \%)$ cases managed in that centre in the 2-year period were reported to IPSU

Conclusion It was difficult to determine the true incidence of $\mathrm{CDH}$ using the IPSU data due under-reporting. Increased reporting may be achieved by raising awareness and by the use of capturerecapture mechanism. The establishment of a national congenital anomaly register is also indicated.

\section{THREE SIBLINGS WITH NEONATAL PRESENTATION OF GRISCELLI SYNDROME}

doi:10.1136/archdischild-2012-302724.0614

BR Oandalji. Pediatrics, Ministry of Health, Amman, Jordan

Introduction Griscelli syndrome type 2 is a rare disorder charecterised by pigment dilution (silvery hair), variable immune deficiency, and tendency to develop a life threatening hemophagocytic syndrome. Presentation in neonatal life is even more rare.

Materials and Methods These are three siblings to first cousin parents who presented with Griscelli syndrome in the neonatal life with dessimel outcome.

Results J, A, S, are three siblings, two sisters and one brother born to first cousin parents. All had normal vaginal full term delivery. All presented on day one of life with pallor, silvery hair, and hepatosplenomegaly. Investigations revealed variable degrees of anemia \& thrombocytopenia. BM revealed hemophagocytic syndrome. There was no HLA matched BM donor so they were treated conservatively. They had repeated admissions for infections and received several blood and platalet transfusion. They had stoem courses and died by two months of age.

Conclusion This is probablly the largest series of Griscelli syndrome presenting in neonatal life. BM is the only hope fore this syndrome.

Athorough family history is always helpfull in diagnosing difficult cases.

\section{CONGENITAL LUNG MALFORMATIONS PRESENTING WITH SIMILAR CHEST X-RAY CHANGES AT BIRTH}

doi:10.1136/archdischild-2012-302724.0615

${ }^{1} \mathrm{G}$ Nepali, $2 \mathrm{M}$ Dattani, 'S Somisetty. 'Neonatal Unit, Luton and Dunstable Hospital NHS Foundation Trust, ${ }^{2}$ Radoiology, Luton \& Dunstable Hospital NHS Foundation Trust, Luton, UK

Congenital defects of diaphragm or malformations of lung usually present in first few hours of birth and if left undiagnosed can lead to significant morbidity and mortality. Early accurate recognition is paramount for subsequent management.

Aim To highlight cases presenting to tertiary neonatal unit with respiratory distress on admission and similar chest x-ray changes.

Case 1 Term infant, one of dichorionic diamniotic twins with antenatal history of polyhydramnios admitted with respiratory distress soon after birth. Initial Chest X-ray (CXR) showing homogeneous opacification of left hemithorax with mediastinal shift to right. Subsequent CT chest revealed Bronchogenic cyst.

Case 2 Term infant admitted with respiratory distress. Initial CXR showed homogeneous opacification of left hemithorax with mediastinal shift to right. Subsequent CXR revealed left sided diaphragmatic hernia.

Case 3 Premature infant born at 34 weeks of gestation with multiple congenital anomalies. Initial CXR showed almost complete opacification of right hemithorax. Chest ultrasound was suggestive of severe right sided diaphragmatic eventration.

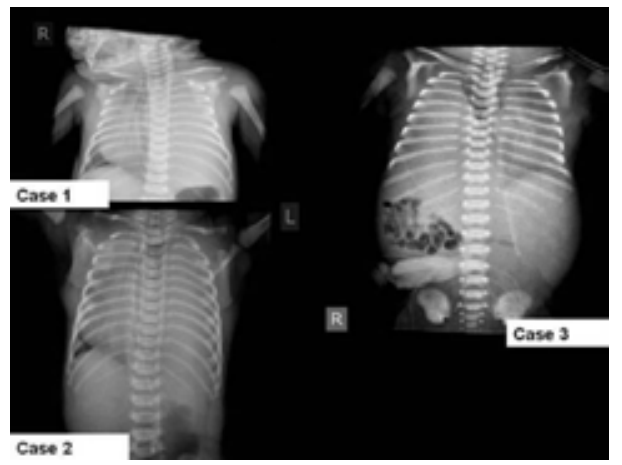

Abstract 615 Figure 1 Initial Chest X-ray 